Fourth International Conference on Semantics, Knowledge and Grid

\title{
Inside the NIGM Grid Service: Implementation, Evaluation and Extension
}

\author{
Peter Brezany, Ibrahim Elsayed, Yuzhang Han, \\ Ivan Janciak, Alexander Wöhrer \\ Institute of Scientific Computing \\ University of Vienna \\ A-1090 Vienna, Austria \\ \{brezany,elsayed,han,janciak,woehrer\}@ par.univie.ac.at
}

\author{
Lenka Novakova, Olga Stepankova, \\ Monika Zakova \\ Department of Cybernetics \\ Czech Technical University in Prague \\ 16627 Prague 6, Czech Republic \\ \{novakova,step,zakovm1\}@labe.felk.cvut.cz
}

Jianguo Han

School of Information Science and Technology

Beijing University of Chemical Technology

Beijing 100029, China

han@mail.buct.edu.cn

\begin{abstract}
Chinese and Western medicine $s$ have a different understanding and approach to life, health, and illness joining their complementary work and support them by an advanced information technology could result in an improved health system. The Non-Invasive Blood Glucose Measurement (NIGM) Service is a grid based implementation of a novel non-invasive method for measuring human blood glucose values exploiting Chinese meridian theory. In this paper, we describe the implementation of the NIGM service in detail, present an initial performance evaluation and discuss an extension towards other non-invasive long term diabetic relevant measurement. Additionally, the adaption of the ontology-based Medical records Annotation Tool (MedAT) framework towards usage in NIGM trails is elaborated.
\end{abstract}

\section{INTRODUCTION}

The complex methodology and models of Traditional Chinese Medicine [1] offer different approaches to diagnose and treat a persons health condition than typical Western medicine. It is important to search for ways how to make this often hidden knowledge explicit and available to a broader audience because mutual synergies between Western and Chinese medicines can result in improved patient care.

Grid computing [2] is the infrastructure of choice to enable e-Science [3] by supporting a collaborative way of working together in a secure and efficient manner. eHealth [4] promises to improve health care by using information and communication technology. The problem of diabetis mellitus [5] is urgent around the world (estimated 100 million [6]). To improve quality of life, minimize short term problems (e.g. unconsciousness, hypoglycemia) and prevent severe mid and long term complications (e.g. damage to nerves and small blood vessels of eyes, kidneys and heart, risk of atherosclerosis) the patients have to monitor their blood sugar levels. This is currently ensured by repetitive invasive blood sugar measurements which are often performed even several times a day and that cause a lot of inconvenience to the diabetic patients.

Our earlier contribution in this area, the Non-Invasive Blood Glucose Measurement (NIGM) Service [7], [8], described the grid based implementation of a novel non-invasive method for measuring human blood glucose values accurately and conveniently by the use of a special medical meridian measurement instrument and the China-Austria Data Grid (CADGrid) infrastructure [9]. The data obtained by this instrument is analyzed by the meridian electro information transmission model [10] to derive human blood glucose values. This model consists of a number of computer intensive algorithms, implemented as a complex grid-based computing process. Data Mining [11] is the convenient extraction of patterns representing knowledge implicitly stored in large volumes of data. It has two highlevel goals, namely prediction and description, and can be considered either as an answer to a sophisticated database query or the result of an execution of a data mining workflow. The first is called the descriptive approach, while the second is the procedural approach. The NIGM-Service follows the procedural approach in order to predict blood sugar values. Splitting the process of vital parameter estimation into an evolvable, personalized data mining process and a rather simple source signal emitting and recording phase has two main benefits: first, the infrastructure can easily be applied to other target values and second, improvements to the overall NIGM process and involved data mining services will directly result in a more precise and robust estimation of target values. In this paper, we describe the internals of the NIGM service in detail and present promising initial performance results. Additionally, a novel model for a non-invasive measurement method of the long term blood sugar indicator value, called $H b A_{1 c}$ [12], is described.

In order to be accepted by the general medical community, 
trials [13] should find out the medical conditions under which the NIGM-predicted blood glucose values are reliable. That is why it will be important to collect a rich dataset containing reliable data about measured persons. The Medical records Annotation Tool (MedAT) [14] provides a framework for semiautomatic semantic annotation and transition of data from written (free text) patient records into database records. The adaption of the MedAT framework towards usage in NIGM trails is elaborated, showing its great potential to be used for various medical applications needing a specialized data acquisition support.

The rest of the paper is organized as follows. Section II describes the implementation of the NIGM service in detail, discusses its extension towards predicting another important long term diabetic measurement and presents an initial performance evaluation. In Section III the adaption of the MedAT framework for NIGM trails is elaborated. We close the paper with an outlook on future work and brief conclusions in Section IV.

\section{NIGM SERVICE DETAILS}

The WEEP Engine [15] provides a run-time environment for service-oriented architectures like the Grid, in which process instantiation and activation occurs, utilizing a set of internal components responsible for interpreting and activating the process definitions and interaction with the external resources necessary to realize the defined activities. The WS-BPEL (Web Services Business Process Execution Language) [16] version 2.0 is used as a formalism to process and execute workflows composed of WS-I and WSRF-compliant web services. Web service interoperability (WS-I) [17] is a standard to promote interoperability amongst the stack of web services specifications. Web Service Resource Framework (WSRF) [18] is a family of specifications for web services which provides a set of operations that web services may implement to become stateful. The basic functionality of WS-BPEL includes validation of process definitions, forming its internal representations, instantiation of the process, its execution and control. The NIGM service is a composite Web service, which represents a scientific workflow expressed in the WS-BPEL language. The interface of the service specifies inputs for the process, e.g. number of iterations, and output it produces, e.g. neural network model in the Predictive Model Markup Language (PMML) [19]. PMML is an XML-based language defined by the Data Mining Group which provides a way for applications to define statistical and data mining models and to share models between PMML compliant applications. Fig. 1 illustrates the overall process presented as a sequence of analytical services used to perform particular activities in the workflow.

The NIGM service consist of the following algorithms, deployed as WS-I and WSRF-compliant CADGrid services: (1) System Identification, (2) Kalman Filtering, (3) Fast Fourier Transformation, (4) Combination Service, and finally (5) Back Propagation Neural Network. Their invocation is depicted in Fig. 1. The first three Services are invoked iteratively within a

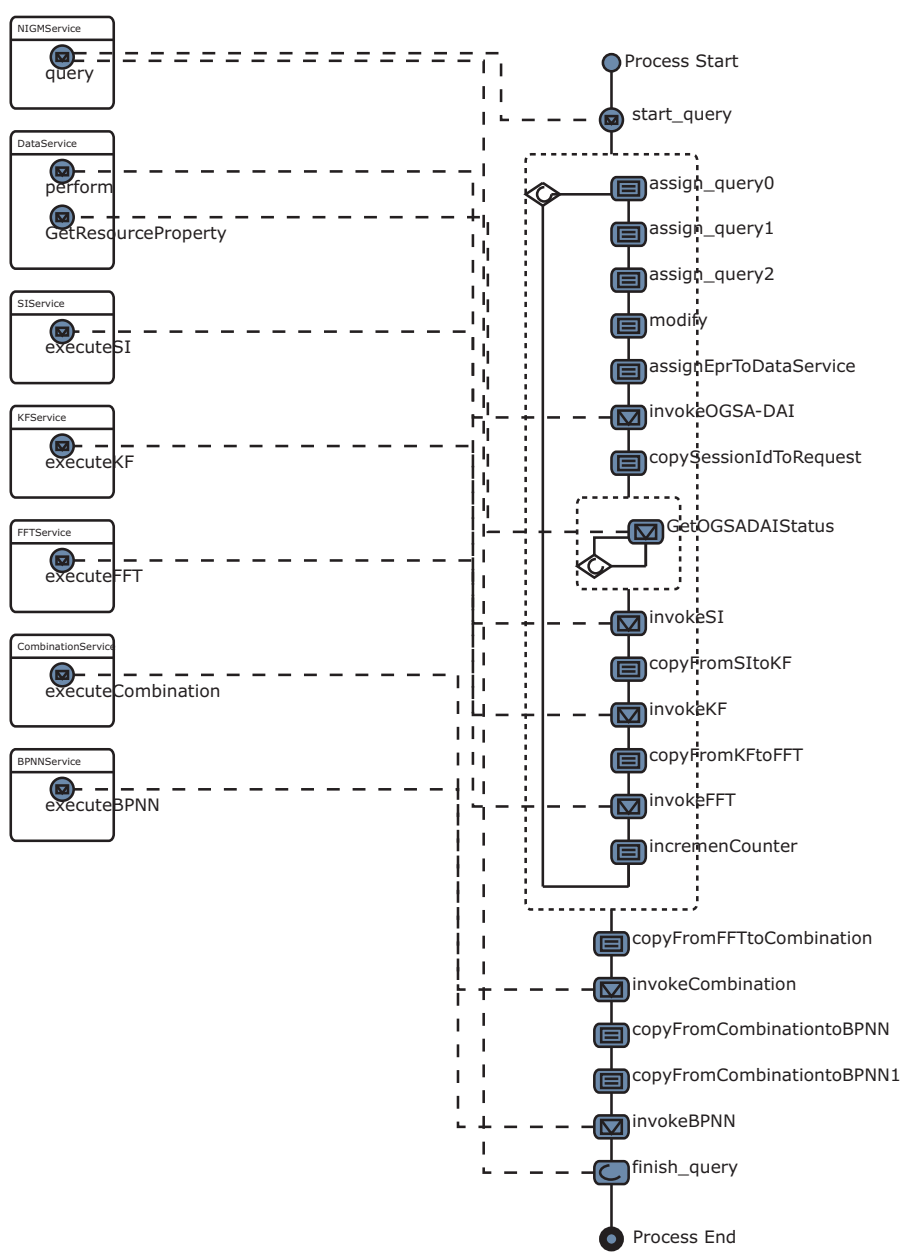

Fig. 1. Graphical representation of Non-Invasive Blood Glucose Measurement (NIGM) workflow specified in WS-BPEL

loop, until all eigenvalues for a given set of input value pairs (meridian measurements) are computed.

At the beginning of each iteration the OGSA-DAI DataService (see the left upper area of Fig. 1) is invoked to retrieve input data for a particular experiment and delivers the data in Java's WebRowSet XML format to the services performing the requested calculations. Open Grid Service Architecture Data Access and Integration (OGSA-DAI) [20] is the de-facto middleware product that allows data resources, such as relational or XML databases, to be accessed via web services. The SIService implements a System Identification [21] algorithm which tries to determine a mathematical relation between the external influences (inputs to the system) and the behavior of it. It receives the files delivered by the DataService and generates the input files used by the KFService. The KFService implements the Kalman Filter [22] algorithm to extract a signal from a series of incomplete and noisy measurements in the data file generated by the SIService, which serves as input for the FFTService. The FFTService implements the Fast Fourier Transformation (FFT) [23] algorithm. The service calculates the eingenvalues from the transformed waveform using FFT. The eingenvalues of all the iterations will be combined to- 


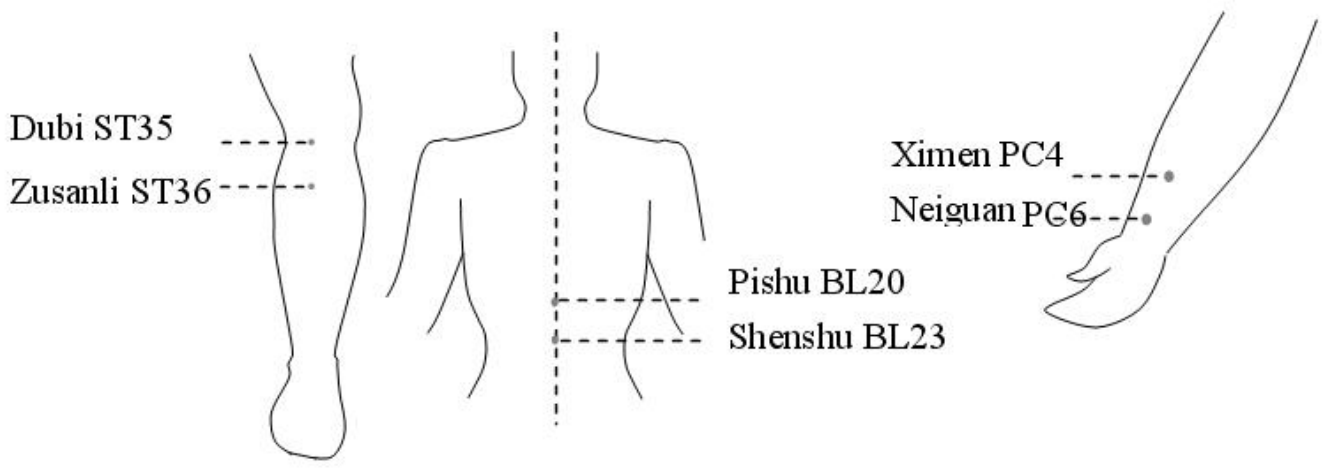

Fig. 2. Acupuncture-points for $H b A_{1 c}$ value prediction: Zusanli ST36 and Dubi ST35 input/output of kidney related meridian-subsection; Shenshu BL23 and Pishu BL20 input/output of liver related meridian-subsection; Neiguan PC6 and Ximen PC4 input/output of blood control system related meridian-subsection.

gether by the CombinationService. The first services of the overall NIGM workflow can be seen as the data preparation phase of our knowledge finding task. The CombinationService is executed after all the iterations. The service combines all the output data files produced by the FFTService together with the file containing the measured blood sugar data. The newly created file is used as an input for the BPNNService. The file provides the input values for the training process of a neural network and supervisor values. The BPNNService has two functions:

1) It builds an individual health model for each considered person. This model is patient specific and has the form of neural net. It is stored in a PMML file.

2) The patient specific model is used to predict blood glucose value for the considered particular patient.

\section{A. Extending NIGM}

Hemoglobin is a substance responsible for carrying oxygen throughout the body. When diabetes is not controlled (meaning that blood glucose is too high), sugar builds up in blood and combines with hemoglobin, that is becoming 'glycated'. The glycohemoglobin or hemoglobin A1c (abbreviated as $H b A_{1 c}$ [12]) provide information about average level of person's blood glucose during the period of last 6 to 12 weeks. The $H b A_{1 c}$ values (obtained currently by a blood test) are used in Western medicine to determine how well diabetes is being controlled and its value is used in conjunction with home blood glucose monitoring to make adjustments e.g. in diabetes medication.

Similar to our original work on predicting blood glucose values via meridian measurements, we elaborate in this section on the extension of the NIGM service with a novel model for the prediction of $H b A_{1 c}$ values on the basis of the electrosignal-transmission-character-values from the kidney related meridian-subsection (KMS), liver related meridian-subsection (LMS), and blood control systems related meridian-subsection (BMS).

A $H b A_{1 c}$ value $(\mathrm{HBV})$ to be measured on time $\mathrm{k}(\mathrm{k}=1,2$, $\ldots$, the time-points with constant interval), marked as $\operatorname{HBV}(\mathrm{k})$, can be forecast by using a time variable back propagation neural-network (BPNN) for its prediction based on the following 13 value-series:

- $\operatorname{HBV}(\mathrm{k}-1), \operatorname{HBV}(\mathrm{k}-2), \mathrm{HBV}(\mathrm{k}-\mathrm{r})$ called $H b A_{1 c}$ value series for time point $\mathrm{k}-1$, marked as vector $\mathrm{HB}(\mathrm{k}-1)$

- CFMS(k), CFMS(k-1), CFMS(k-r) of KMS, LMS and $\mathrm{BMS}$, marked as $\mathrm{KC}(\mathrm{k}), \mathrm{LC}(\mathrm{k}), \mathrm{BC}(\mathrm{k})$ respectively

- $\operatorname{STEE}(\mathrm{k}), \mathrm{STEE}(\mathrm{k}-1), \operatorname{STEE}(\mathrm{k}-\mathrm{r})$ of KMS, LMS and BMS, marked as $\mathrm{KS}(\mathrm{k}), \mathrm{LS}(\mathrm{k}), \mathrm{BS}(\mathrm{k})$ respectively

- MTPD(k), MTPD(k-1), MTPD(k-r), of KMS, LMS and BMS, marked as $\mathrm{KM}(\mathrm{k}), \mathrm{LM}(\mathrm{k}), \mathrm{BM}(\mathrm{k})$ respectively

- DTFB(k), CTFB(k-1), CTFB(k-r) of KMS, LMS and $\mathrm{BMS}$, marked as $\mathrm{KD}(\mathrm{k}), \mathrm{LD}(\mathrm{k}), \mathrm{BD}(\mathrm{k})$ respectively

The used functions are defined as follows:

- CFMS: Correlation Factor between Input-, Output-Signal of Meridian-Subsection

- STEE: Sum of Transmission-Electro-Energy

- MTPD: Maximal Transmission Power Density

- DTFB: Distribution center of Transmission Frequency Band

- $r$ : the time point value to be decided trough experiment.

The data series mentioned above are combined totally into a matrix called meridian information matrix for time point $\mathrm{k}$, marked as $\operatorname{MIX}(\mathrm{k})$. The overall workflow for this extended version of NIGM for predicting $H b A_{1 c}$ looks nearly the same as for the short term blood glucose values, pictured in Fig. 1, except the fact that the input matrix for the BPNNService is larger than before. This reflects the fact that three sets of meridian measurement data, from the six meridian points pictured in Fig. 2, are needed to predict $H b A_{1 c}$ values compared to one set for blood glucose values.

Reasons of AC-point's selection. For the short term blood glucose measurement NIGM service, the selected ac-points, 'Tai Chong' and 'Tai Xi' belonged to two meridians related to the kidney and the liver respectively. The consideration of this is to sense the reactions of two meridians using only one crossing-sub-section conveniently, but comprehensively. For $H b A_{1 c}$ prediction, the meridian-sub-sections pictured in Fig. 2 were selected. This respects the fact that the middle/longterm reflection of meridians to the diabetes through $H b A_{1 c}$ 


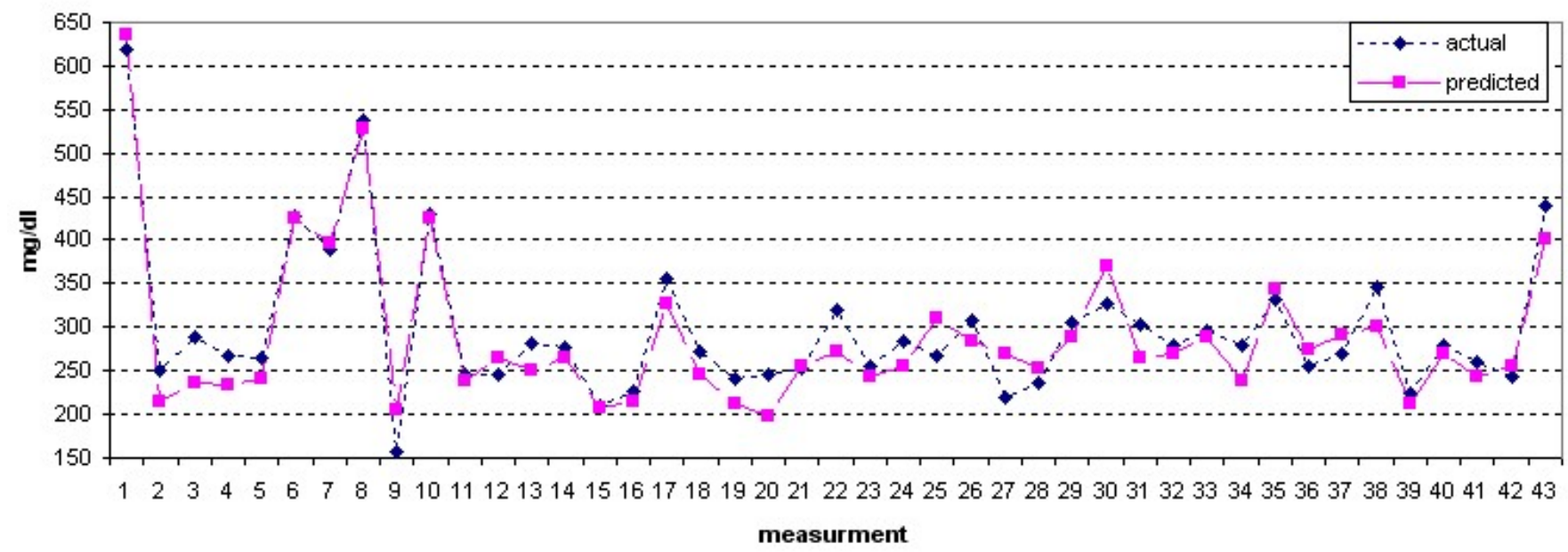

Fig. 3. Actual measured blood glucose values in the evaluation dataset and corresponding predicted values by an individual trained model via NIGM.

values should be more complete and synthesized related to the total body system mainly on kidney-, liver-, and bloodsystem. Dubi ST35-Zusanli ST35, Shenshu BL23-Pishu BL20, Neiguan PC6-Ximen PC4 effect and reflect the health situation of the kidney, the liver and the blood system very strongly. The health situation of the three organs effect and reflect the longterm illness situation of diabetes strongly, resulting in the values of $H b A_{1 c}$. Of cause, there is also very strong relationship between the measured and calculated values mentioned above (but $H b A_{1 c}$ is not needed) and other illnesses like high/low blood-pressure, hepatitis, nephritis, etc.

Usage of other body-parameters. Not only meridian measurement data mentioned above can be used to support the $H b A_{1 c}$ prediction but also other body parameters. For example, the values of weights recorded at time k, k-1, k-r (WE(k), WE(k-1), WE(k-r)), the average eat-in-values (united in $\mathrm{mg}$ ) of special physical-/chemical- materials such as sugar, salt, fat recorded at time $\mathrm{k}(\mathrm{SUV}(\mathrm{k}), \mathrm{SAV}(\mathrm{k}), \mathrm{FAV}(\mathrm{k}), \mathrm{SUV}(\mathrm{k}-$ 1), SAV(k-1), FAV(k-1), , SUV(k-r), SAV(k-r), FAV(k)-r) can be used and combined into the MIX(k) for improved $H b A_{1 c}$ prediction.

\section{B. NIGM Evaluation}

The following setup was used for our initial performance evaluation of the NIGM workflow pictured in Fig. 1. All services are hosted on a Linux server running Fedore Core 5 on an Intel Pentium D $930 @ 3 \mathrm{GHz}$ with 4 GB of RAM. The following software versions are used: Globus Toolkit 4.05 [24], WEEP Version 1.2.1 [15], OGSA-DAI WSRF 2.2 [20] exposing a MySQL 4 database with the meridian measurement test datasets collected over several weeks with the earlier mentioned meridian measurement instrument. Currently, it takes around 1 minute to obtain one set of meridian measurement data. The training dataset consists of 129 pairs of meridian measurement data and corresponding blood glucose value from one patient used to train an initial individual blood glucose prediction model for him. The times needed to train and use the model are given in Table I. The most expensive parts of the overall blood glucose modeling process are system identification and the overheads related to service oriented computing and workflow execution.

Initial model creation (training phase) is defined by the sum of all data preparations (equivalent to the sum of 'time of one loop' in Table I) plus combination of the resulting values plus BPNN training, resulting for our model creation in

$$
126+0,8+30=156.8 \text { seconds }
$$

Model maintenance time $t$ is dependent on $n$, the number of new pairs of meridian measurement data and corresponding blood glucose value.

$$
t=n *(l+x+y) \text { seconds }
$$

In the function above, $l$ is the time needed for preparing one meridian measurement dataset (equivalent to the average of 'time of one loop' in Table I); $x$ represents the time needed for the combination service (typically $<0.1 \mathrm{sec}$ ); and $y$ represents the time needed for maintaining the BPNN (using the current model as initial starting point, typically $<1 \mathrm{sec}$ ).

\begin{tabular}{|l|c|c|c|c|}
\hline Service & $\min$ & $\max$ & avg & sum \\
\hline \hline OGSA-DAI & 78 & 805 & 155 & 19.998 \\
\hline SI & 150 & 409 & 173 & 22.323 \\
\hline KF & 68 & 358 & 101 & 12.970 \\
\hline FFT & 107 & 391 & 147 & 18.900 \\
\hline Overheads & 123 & 984 & 404 & 52.235 \\
\hline \hline time of one loop & 640 & 2102 & 980 & 126.426 \\
\hline \hline Combination & 758 & 758 & 758 & - \\
\hline BPNN training & 30.003 & 30.003 & 30.003 & - \\
\hline \hline BPNN usage & 98 & 162 & 150 & - \\
\hline
\end{tabular}

TABLE I

PERFormanCE OF EACH SERVICE USED B Y NIGM IN MS. 
Blood glucose prediction with the current model (model usage) takes

$$
1+0,15=1.15 \text { seconds }
$$

It should be mentioned that the times of the services in the loop needed for data preparation (SI, KF, FFT) are heavily dependent on the sampling size used to analyze the meridian measurement data. Each meridian measurement data set used in the above evaluation had the size of $100 \mathrm{~KB}$, containing 1.000 rows. Bigger data sets up to $1 \mathrm{MB}$ each, containing 10.000 rows, are possible. The effects (and possible correlations) of the sampling size on the model accuracy are targets in forthcoming more detailed evaluations.

\begin{tabular}{|l|c|c|c|c|}
\hline relative deviation & $<5 \%$ & $5-10 \%$ & $10-15 \%$ & $>15 \%$ \\
\hline \hline number & 14 & 15 & 9 & 5 \\
\hline percentage & $33 \%$ & $35 \%$ & $20 \%$ & $12 \%$ \\
\hline
\end{tabular}

TABLE II

STATISTICS OF INITIAL INDIVIDUAL PREDICTION MODEL.

The evaluation dataset consists of 43 pairs of meridian measurement data and corresponding blood glucose value. Statistics about the accuracy of the initial trained individual blood glucose prediction model on this new data can be found in Table II The predicted values were compared with measured ones, see Fig. 3, resulting in $88 \%$ of the predicted values possessing relative deviations ranging within $+/-15 \%$. The overall mean of relative deviations for the evaluation dataset is $8.71 \%$. Standard deviation amounts 0.06541 .

Typical modern invasive blood glucose measurement devices need below 15 seconds to display a value within +/$10 \%$ to $+/-15 \%$ accuracy when used optimally, depending on the device used.

In [8], the performance of a FPGA [25] based implementation of a one non-individual blood glucose prediction model built for a group of 300 patients is discussed. The predicted values were compared with measured ones, resulting in $75 \%$ of the predicted values possessing relative deviations ranging within $+/-15 \%$, showing a small effective area of a single non-individual prediction model for a group of patients.

\section{Medical Records Annotation Tool}

The NIGM-Service offers a novel promising non-invasive technology for measuring patient's data. In order to be accepted by general medical community, the quality of provided results will have to be verified in extensive clinical trials following a well defined protocol [26]. The main aim of these trials should be to specify medical conditions under which values of blood glucose and of $H b A_{1 c}$ obtained through NIGM-Service are as reliable as data provided by the invasive measurement techniques that are currently the only standard used and accepted by Western medicine.

That is why it will be important to collect a rich dataset of authentic sound data about the persons participating in the trials that will not be limited to time-stamped results of both non-invasive and invasive measurements taken, but that will also contain additional background information about the participant's health condition, e.g.:

- anamnestic data including year of birth, sex, weight, height, blood pressure, etc.,

- lifestyle data describing participant's average physical activity level per day, dietary habits, smoking habits, etc.,

- presence of various risk factors including heart and kidney disease, neuropathy or retinopathy,

- list of participant's current medication,

- more details about his/her diabetic condition (if the person is diagnosed as a diabetic patient or not, if so what type of diabetes has been identified, ..).

A detailed analysis of the collected data will be necessary to demonstrate that the testimony provided by the clinical trial is not biased towards age, sex, specific diseases, etc. Moreover, the collected data should be anonymised and stored for further usage because it can become a valuable and useful source for other data mining studies aiming at further improvements in diabetic care resulting in higher patient comfort.

Creation of such a dataset would be significantly simplified if all the treated patients had their electronic health record (EHR) [27]. In such a case we could rely on the data collection facilities provided by NIGM-Service and complement it later by data from EHR. But we are still far from the time when this can be achieved. For example currently in the USA, EHR is available for just a small fraction of citizens (5\%). That is why it is necessary to integrate the NIGM-Service with a facility that will help to create a unified framework for transfer of relevant data from written (free text) patient records into a database format. To reach this goal we suggest to tailor the MedAT [14] tool towards this application.

Medical records Annotation Tool (MedAT) [14] provides a framework for semi-automatic semantic annotation of information which is not currently available in an explicit form and which cannot be employed in the data-mining analysis consequently. MedAT expects its input in the form of a textual report (or as a part of complex multimedia patient's record) and it helps to transform this input into machineunderstandable format using OWL [28] formalism (instances of the resulting knowledge base can be easily exported to a relational database). Currently, MedAT provides three types of services ( 1 and 2 are fully implemented, the last one is under development):

1) user-friendly form-like interface for entering patient data,

2) creation and maintenance of a central relational database (RD) for storing the data collected from distributed sources,

3) sophisticated data preprocessing and understandable graphical presentation of results obtained by on-line analysis of data in the RD that provides basic information necessary for understanding the collected data. 


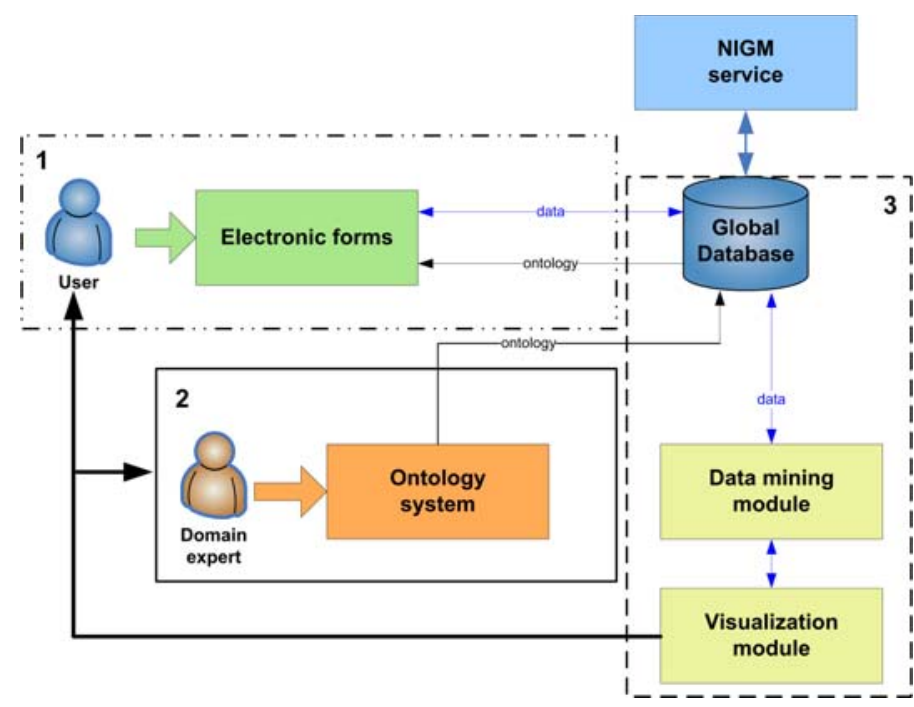

Fig. 4. MedAT structure and data-flow of the joint NIGM+MedAT system.

These three types of services are depicted in the Fig. 4 each service in a separate frame denoted by the corresponding number. The forms used in the step 1 (see Fig. 5 for a simple example) can be further refined during the data collection process according to the requirements of the users, ie. medical experts, based on the results of on-line data analysis mentioned in the above item 3. This refinement can be ensured simultaneously at all nodes of data-collection grid and the corresponding change is reflected in item 2 (RD maintenance) so that data consistency is not endangered. The current version of MedAT has been successfully applied for medical data collection and analysis in several wards in Czech hospitals (genetics, children's and adult neurology, etc.) and it was well accepted by its users - medical experts, who acknowledge its user-friendliness.

The annotation process is ontology driven and thus it is not limited to a single specific type of patient record. It proceeds through filling of forms containing concepts relevant to a particular patient record or through editing data in this form provided that initial data have been automatically loaded from another structured source, for example from a database table. The forms are introduced as the user interface, since they are close to database applications that are very frequent in medical domain and that are already well accepted by medical community. The resulting annotations are represented in OWL formalism.

MedAT is based on 2 types of ontologies:

- a domain ontology defining the concepts and properties, which are to be annotated,

- an ontology describing the GUI elements of MedAT forms such as text fields, drop-down lists, radio buttons, etc.

In the domain ontology, the concepts and properties relevant for a particular considered task are described, e.g., a disease and its characteristic symptoms, examinations and treatment. MedAT applies Jena API for dealing with ontologies [28].
Consequently, MedAT is ready to utilize a subset of any public medical ontology, it can work with its own ontology designed in cooperation with domain experts for the specific application or both approaches can be combined.

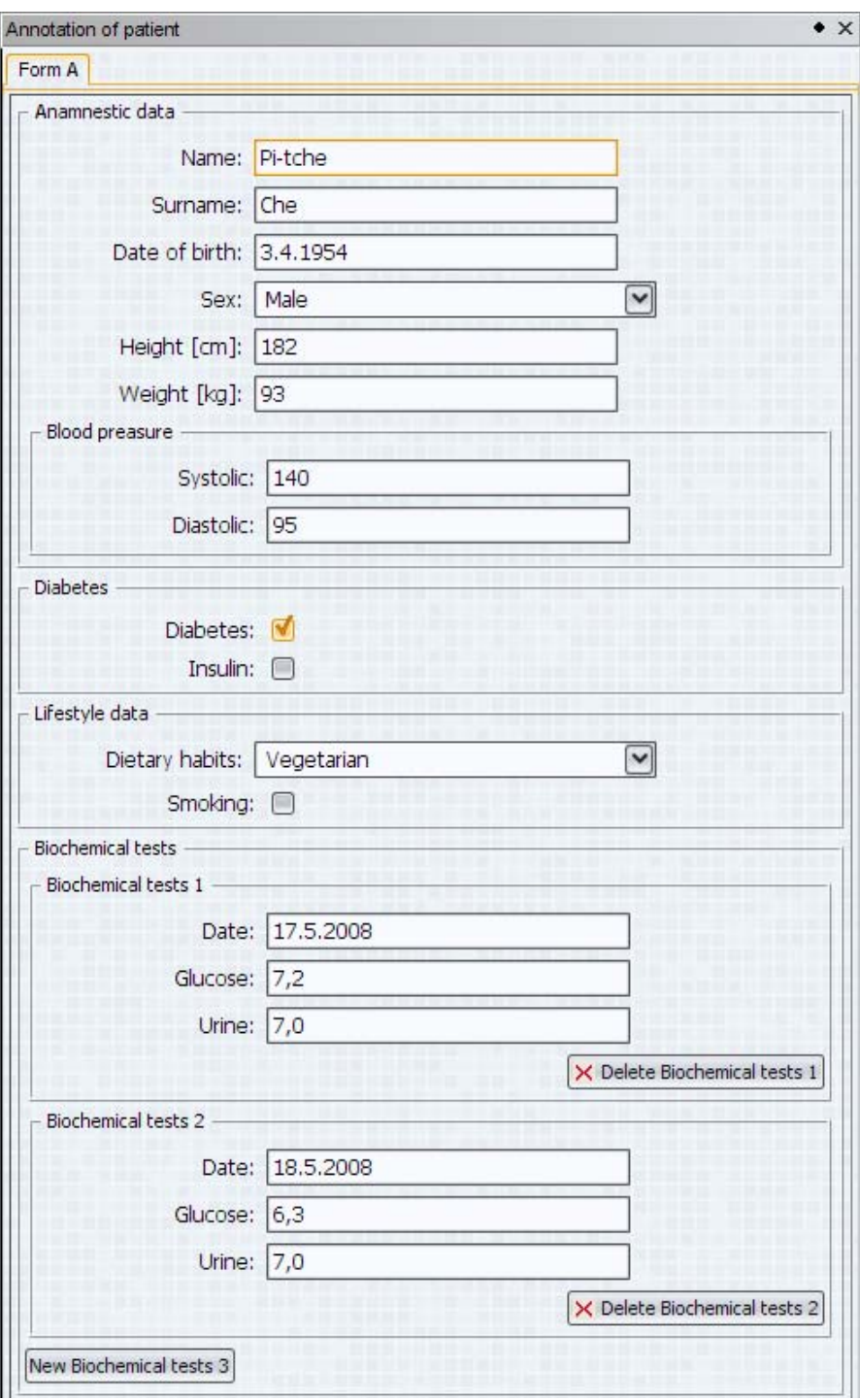

Fig. 5. Patient's medical form in MedAT

To enable automatic creation of necessary forms, a simple MedAT GUI ontology describing the representation of particular concepts in the form has been defined. The concepts from the domain ontology are mapped on the concepts from MedAT GUI ontology by creating a subontology of both considered ontologies and adding subclass $O f$ relationships stating which domain concepts are subclasses of the concepts from the MedAT GUI ontology. The subclass Of relationship was used instead of range to avoid introducing relations concerning GUI, such as relation displayedAs, into the domain ontology. MedAT takes this newly created ontology as its input and generates automatically electronic forms, which are to be filled in by the medical expert. A simple example of a form used within future NIGM trails is depicted in Fig. 5. 
The important advantage of the electronic form is its dynamic structure - the user (e.g. medical expert) can interactively modify content of the predefined part of the used form, see the button named "New Biochemical test 3" in Fig. 5. The reverse operation, i.e. deletion, is available, too - see the buttons named "Delete Biochemical test 1" and "Delete Biochemical test 2". These two buttons ensure dynamic behavior of the form representing 1:N relation. Thanks to this feature MedAT is ready to collect and store highly relational data about participants included in he study. This is a must in case of longitudinal studies because number of repetitive measurements concerning individual participants can vary significantly: it happens often that one patient inputs his/her measurements for several days while another one for several weeks.

To analyze the resulting relational data sophisticated datapreprocessing has to be applied. Services provided by MedAT currently support some of these tasks as a part of service no. 3 mentioned earlier, see the Fig. 4). At present, a further extension of the available services is being planned, this augmentation will include propositionalization of the collected relational data.

Technologies used in design and implementation of MedAT are compatible with those used in the CADGrid infrastructure and both systems seem to be well suited for smooth cooperation. The resulting system will be able to support complex evaluation of meridian measurements by NIGM-Services as well as all processes related to collection of complementary data about individual patients and to their on-line analysis through the MedAT services.

\section{Conclusions}

The NIGM grid service combined with the MedAT system for semantic annotation of measured data will be able to build a unique dataset containing valuable information related to various characteristics of diabetic patients including time development of blood glucose level and meridian measurements as well as the individual prediction models created by the NIGM services. Extensive datamining is planned for the resulting dataset. It is expected that the Knowledge Grid [29] will be used as an advanced future infrastructure for enhancing the collected data with intention to build generic prediction models which can be further reused.

The research effort described in this paper details a promising marriage between a combination of Western and Chinese medicine and advanced information technology. The internals of the current Non-invasive Blood Glucose Measurement (NIGM) service are presented together with promising initial performance results. Additionally, an extension to it via a novel model for a non-invasive measurement method of the long term blood sugar indicator value $H b A_{1 c}$ was introduced. The adaption of the MedAT framework towards usage in NIGM trails, needed in order to be accepted by the general medical community, was elaborated. This shows MedAT's great potential to be used for various medical applications needing a specialized data acquisition support.

The original NIGM-Service expects that the patient's model is being created or updated quite often. Obviously, this approach is necessary in the research phase of the system when the main goal is to provide arguments that the new tool and its methodology is reliable and works well. But this solution demands a lot of resources and consequently must be rather expensive. To make the suggested NIGM solution accessible to vast amount of those who need it, it is necessary to search for methods that can make the necessary data processing "cheaper" and less dependent on the sophisticated Grid technology (which does not have to be available at every place where the treated patient goes or stays). That is why it is important to search for frequently used types or clusters of models which can be considered as generic ones. The data collected in the relational database mentioned above should be carefully analyzed in order to specify those properties which allow for "re-use" of such models (in the same way as in the case of pacemakers).

It will be most interesting to study similarities among various models created by NIGM-Service for different persons. If these models form some clusters it will be important to answer following question "Can the membership of the individual model to a certain cluster be determined from data stored in EHR and/or one shot measurement of person's meridian values?" If this appears to be the case at least for a certain type of patients, the NIGM-Service could enter a similar development track as pacemakers regulating the rate that the heart beats. Currently pacemakers are produced in 3 basic types differing by number of leads. The medical expert chooses the best type of pacemaker for the considered patient and its function is customized to the patient's individual requirements by setting the parameters during the installation. In the case of NIGM-Service, this approach would mean that the user could rely on his/her own individualized non-invasive blood glucose measurement system that is able to work offline from Internet connection for certain period of time while returning back to the grid service for necessary calibration of this system in well established periods.

This could lead not only to improvement of patient's quality of life but also to significant reduction of price of the relevant service.

\section{ACKNOWLEDGMENT}

The work described in this paper is being carried out as part of the bilateral Austrian-China project "Medical Measurement Grid for On-Line Diagnosis" and "Austrian Grid" supported by the Austrian Federal Ministry of Science and Research. The work is also supported by AV-CR 1ET 101210513 "Relational machine learning for analysis of biomedical data" and by the bilateral Austrian-Czech project "Advanced Data Management Strategies for the On-Line Diagnosis Grid". 


\section{REFERENCES}

[1] G. Maciocia, The Foundations of Chinese Medicine: A Comprehensive Text for Acupuncturists and Medical Herbalist. Elsevier Churchill Livingstone, 2005.

[2] D. De Roure, M. A. Baker, N. R. Jennings, and N. R. Shadbolt, "The evolution of the grid," in Grid Computing: Making The Global Infrastructure a Reality, F. Berman, A. J. G. Hey, and G. Fox, Eds. John Wiley \& Sons, 2003, pp. 65-100.

[3] A. Krishnan, "A survey of life sciences applications on the grid," New Generation Computing, vol. 22, no. 2, pp. 111-126, 2004.

[4] S. Martin, D. C. Yen, and J. K. Tan, "E-health: impacts of internet technologies on various healthcare and services sectors," International Journal of Healthcare Technology and Management, vol. 4, no. 1/2, pp. 71-86, 2002.

[5] WHO-IDF, "Definition and diagnosis of diabetes mellitus and intermediate hyperglycemia," Report of a WHO/IDF Consultation, 2006.

[6] N. A. Bazaev and S. V. Selishchev, "Noninvasive methods for blood glucose measurement," Biomedical Engineering, vol. 41, no. 1, pp. 42 50, 2007.

[7] I. Elsayed, J. Han, T. Liu, A. Woehrer, F. A. Khan, and P. Brezany, "Gridenabled non-invasive blood glucose measurement," in International Conference on Computational Science (ICCS), 2008.

[8] H. Cao, D. Ma, X. Wang, Y. Han, and J. Han, "A novel non-invasive medical measurement based on parallel neural computation supported by the grid," in International Conference on Natural Computation (ICNC), 2008.

[9] CADGrid, "China-Austria Data Grid," http://www.par.univie.ac.at/ project/cadgrid/, 2007.

[10] J. Han, Y. Han, Q. Xia, X. Hou, and Y. Li, "Experiment and analysis on transmission-characteristics of human-body-meridians-electroinformation with application," Modern Chinese- and west-Medicine Magazine, vol. 3, no. 19, pp. 17-29, 2005.

[11] J. Han and M. Kamber, Data Mining: Concepts and Techniques. Morgan Kaufmann, 2000

[12] S. M. Marshall and J. H. Barth, "Standardization of $H b A_{1 c}$ measurements - a consensus statement," Diabetic Medicine, vol. 17, no. 1, pp. $5-6,2000$.

[13] S. Chow and J. Liu, Design and Analysis of Clinical Trials : Concepts and Methodologies. Wiley-Interscience, 2004.

[14] M. Žáková, L. Nováková, O. Štěpánková, and T. Maříková, "Ontologies in Annotation and Analysis of Medical Records," in Distributed Human Machine Systems. Praha: CTU Publishing House, 2008, pp. 198-203.

[15] I. Janciak, C. Kloner, and P. Brezany, "Workflow enactment engine for wsrf-compliant services orchestration," in The 9th IEEE/ACM International Conference on Grid Computing, 2008.

[16] The OASIS Committee, "Web Services Business Process Execution Language (WS-BPEL) Version 2.0," http://www.oasisopen.org/specs/index.php\#wsbpelv2.0, April 2007.

[17] Web Services-Interoperability Organization, http://www.ws-i.org/.

[18] OASIS, "Web Services Resource Framework," www.oasisopen.org/committees/wsrf/.

[19] Data Mining Group, "Predictive Model Markup Language," http://www.dmg.org/pmml-v3-0.html

[20] OGSA-DAI, http://www.ogsadai.org.uk/.

[21] L. Ljung, System Identification Theory For the User. Prentice Hall, 1999.

[22] R. E. Kalman, "A new approach to linear filtering and prediction problems," 1960.

[23] S. Bochner and K. Chandrasekharan, Fourier Transforms. Princeton Book Comp. Publ., 2001.

[24] Globus Alliance, "The Globus Toolkit," http://www.globus.org/.

[25] S. Kilts, Advanced FPGA Design: Architecture, Implementation, and Optimization. IEEE Computer Society Press, 2007.

[26] S. Pocock, Clinical Trials: A Practical Approach. John Wiley \& Sons, 2004.

[27] G. F. Murphy, M. A. Hanken, and K. A. Waters, Electronic Health Records: Changing the Vision. Saunders, 1999.

[28] A. Gomez-Perez and O. Corcho, "Ontology languages for the semantic web," IEEE Intelligent Systems, vol. 17, no. 1, pp. 54-59, 2002.

[29] H. Zhuge, The Knowledge Grid. World Scientific Publishing Co. Singapore, 2004. 